\title{
Bisfosfonatos en cirugía oral: Bevisión de literatura.
}

Bisphosphonates in Oral Surgery: Literature Review.

Karen Caballero ${ }^{1, a}$, María del Pilar Alvarez ${ }^{l, a}$, Karina Centurión ${ }^{l, a}$, Ghajayra Reyes ${ }^{1, a}$, Marco Alarcón-Palacios $^{2, b}$.

\section{RESUMEN}

Los Bisfosfonatos Orales son medicamentos indicados generalmente para pacientes con trastornos metabólicos siendo hoy en día su uso muy común por ser parte del tratamiento en pacientes diagnosticados con Osteoporosis. La odontología, especialmente en su área de cirugía oral debe conocer el manejo de los pacientes medicados con bisfosfonatos porque en casos particulares puêde llevar a una necrosis ósea que puede condicionar el éxito de nuestro tratamiento. El presente artículo busca hacer una revisión de las propiedades, tipos y relación con los tratamientos odontológicos de los Bisfosfonatos Orales.

\section{Palabras clave: BISFOSFONATOS, CIRUGÍA BUCAL, OSTEOPOROSIS, ALENDRONATO, OSTEONECROSIS.}

\section{ABSTRACT}

Oral bisphosphonates are drugs generally indicated for patients with metabolic disorders, today its common use it in the treatment of patients diagnosed with Osteoporosis. Dentistry, especially in the area of oral surgery should know the management of patients taking bisphosphonates because in particular cases can lead to bone necrosis that may determine the success of our treatment. This article offers a review of properties, types, and relation to dental treatment of Oral Bisphosphonates.

Key Words: BISPHOSPHONATES, BONE, ORAL SURGERY, OSTEOPOROSIS, OSTEONECROSIS.

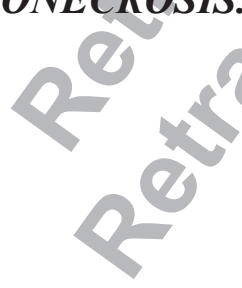

\footnotetext{
${ }^{1}$ Facultad de Estomatología Universidad Privada Antenor Orrego. Trujillo, Perú.

2 Facultad de Estomatología. Universidad Peruana Cayetano Heredia. Lima, Perú.

a Bachiller en Estomatología. Maestranda en Estomatología.

b Magíster en Estomatología. Docente Postgrado Periodoncia e Implantología Oral.
} 


\section{INTRODUCCIÓN}

La osteoporosis es una condición sistémica con alta prevalencia que es tratada desde hace años atrás con bifosfonatos. La literatura odontológica ha reportado varios casos referidos a osteonecrosis de los maxilares en pacientes tratados quirúrgicamente que estaban consumiendo tales medicamentos. Se observó que el pirofosfato, la forma más simple del fosfato era hidrolizado por las fosfatasas a nivel intestinal y por tanto permanecían inactivos cuando se administraban vía oral (VO) y obligaban a su administración por vía intravenosa (IV). Esto llevó a estudiar análogos, que tuvieron características fisicoquímicas similares pero que fueron resistentes a la hidrólisis y no fueron degradados metabólicamente (1). Así, se llegó a los bifosfonatos, análogos sintéticos del pirofosfato endógeno del que se diferencian por presentar un enlace $\mathrm{P}-\mathrm{C}-\mathrm{P}$ en lugar de P-O-P $(2,3,4)$, que los hace resistentes a las fosfatasas y por tanto, más estables y con gran afinidad por los cristales de hidroxiapatita óseos.

Los BF se caracterizan por su baja absorción intestinal (1-5\% de la dosis), pero con una elevada captación y retención a nivel óseo. Aproximadamente en tre el $20 \%$ y el $80 \%$ es captado por el hueso y el resto eliminado por el riñón. Su vida media plasmática es de una hora, pero su permanencia una vez captada por el hueso es de años, ya que los BF se liberan cuando el hueso es reabsorbido $(1,2)$.

Los BF fueron sintetizados en el siglo XIX, sin embargo en 1960 fue introducido el primer bifosfonato en el mercado con fines terapéuticos, el etidronato, potente antimineralizante usado para el tratamiento de las calcificaciones hipertróficas y para limitar el exceso de producción ósea en la enfermedad de Paget $(4,5)$.

En 1995 se reporta el primer caso de una falla de osteointegración en la colocación de implantes en un paciente bajo terapia con bifosfonatos para el tratamiento de osteoporosis. En el año 2001 se empezaron a reportar con más regularidad los efectos secundarios del medicamento, ya que para este momento ya se había difundido ampliamente su uso, y se empezaron a diagnosticar lesiones óseas dentofaciales con el diagnóstico de osteomielitis refractarias; ya en el 2003 se estableció el diagnóstico definitivo de necrosis ósea de los maxilares asociada a los bifosfonatos siendo desde entonces un tema de gran importancia $(4,5)$.

\section{PROPIEDADES BIOLÓGICAS Y MECANIS-} MOS DE ACCIÓN

Capote y cols (2011) reportan las siguientes propiedades y mecanismos de acción de los bisfosfonatos: “1) inhibición de la reabsorción ósea osteoclástica; 2) inducción de los osteoblastos a secretar un inhibidor de la secreción ósea mediada por osteoclastos; 3) inducción de apoptosis en los osteoclastos; 4) inhibición de la apoptosis de osteocitos y células de la linea osteoblástica, y 5 ) efecto antiangiogénico que reduce el EGF (factor de crecimiento endotelial). También presentan propiedades antitumorales: inhibición de la proliferación e invasión de las células tumorales; inhibición de la adhesión de las células tumorales al tejido óseo; inducción de apoptosis en varias líneas celulares malignas e inhibición de la actividad proteolitica de las metaloproteinasas de la matriz".

Se emplean bisfosfonatos en el tratamiento de lesiones osteolíticas en el mieloma múltiple, en metástasis óseas predominantemente líticas (especialmente en el cáncer de mama y de próstata) y en la hipercalcemia maligna inducida por tumores; en el tratamiento de osteoporosis: en pos menopausia, en hombres y en las inducidas por glucocorticoides. Tienen asimismo indicación terapéutica en la enfermedad de Paget, osteogénesis imperfecta y otras enfermedades metabólicas óseas $(2,3,5)$.

\section{TIPOS DE BIFOSFONATOS}

Se agrupan según la vía de administración que se emplee y se clasifican según su mecanismo de acción en dos grandes grupos: A) No nitrogenados, estructuralmente semejantes al pirofosfato, para su acción deben ingresar en la célula, donde al acumularse inhiben ciertas enzimas intracelulares, con efectos deletéreos en la función y sobrevida del osteoclasto. B) Nitrogenados ejercen su acción inhibiendo enzimas de la vía del mevalonato, impidiendo de esta manera la biosíntesis de compuestos isoprenoides, esenciales en la regulación de procesos celulares influyentes en la función y sobrevida del osteoclasto $(1,7)$. 
Se han descrito casos de ulceraciones orales asociados generalmente a una inadecuada toma de BF orales. Algunos de ellos correspondientes a mujeres con osteoporosis posmenopáusica que tomaban alendronato que cedieron con corticoides tópicos $(3,6)$.

\section{OSTEONECROSIS DE LOS MAXILARES ASOCIADA A USO DE BIFOSFONATOS}

La osteonecrosis de los maxilares fue definida como "área de hueso en la región máxilo-facial que permanece expuesta al menos ocho semanas, siempre que los pacientes hayan sido prescriptos con bifosfonatos en ausencia de terapia radiante" $(8,9,10)$.

Histológicamente muestran pérdida del ondulado normal de las lagunas de Howship en su lado resortivo, alejándose de la superficie ósea permitiendo que el osteoclasto muera. Se observan también canales vasculares intactos, aun en áreas con infiltrados inflamatorios y también sobrecrecimiento bacteriano (5).

Se sabe que las zonas que están sometidas a una mayor compresión tienen por este estímulo un mayor recambio óseo, lo que las hace vulnerables al efecto antiosteoclástico de los BF (5).

La tasa de recambio a nivel mandibular es en la cresta alveolar 2 veces más que en la zoná del canal $\mathrm{y}$, de 3 a 5 veces más que en el borde basal. El hueso cortical de la mandíbula es más grueso en la región premolar y molar, y es frecuentemente afectado por la osteonecrosis $(5,11)$.La susceptibilidad de los maxilares a sufrir osteonecrosis puede deberse a que la tasa de recambio del hueso alveolar es 10 veces mayor que en los huesos largos (5).

Los factores de riesgo para la ocurrencia de osteonecrosis se agrupan en cuatro categorías: Factor de riesgo de la propia droga, Factor de riesgo local, Factor de riesgo demográfico/sistémico y Factor de riesgo genético.

Escobar y Cols. (2) en el año 2007, realizaron una revisión sistemática sobre Osteonecrosis de los maxilares asociada a bifosfonatos, en el cual se evaluaron 340 casos. Se encontró que los pacientes presentan como diagnóstico primario principalmente: mieloma múltiple $(51,2 \%)$, cáncer de mama $(31,4 \%)$, cáncer de próstata $(7,1 \%)$ y osteoporosis $(4,1 \%)$. La mandíbula resulta más afectada que el maxilar, conel $59,1 \%$. La relación entre género femenino y masculino es de $2: 2,6$. El pamidronato es el bifosfonato más asociado a los casos de osteonecrosis $(35,3 \%)$.

Prado y Cols. (5) en el año 2011, realizaron un artículo de revisión sobre el manejo estomatológico del paciente en terapia con bifosfonatos. Encontraron una prevalencia de OMIB de 1 al $5 \%$ con terapia de bifosfonatos vía IV, y de 0.001 al $0.01 \%$ con bifosfonatos orales, así como de un 0.09 a $0.34 \%$ después de un tratamiento dental invasivo. Las estructuras más afectadas son la mandíbula en un $78 \%$, el maxilar $16 \%$ y ambos pueden estar afectados en un $5 \%$. Un $52 \%$ en pacientes con antecedentes de extracción dental y el $48 \%$ de una forma espontánea.

Es importante señalar que el riesgo para desarrollar esta complicación aumentará con elempleo de BF nitrogenados, los cuales son muy resistentes a la ruptura hidrolítica (13).

El diagnóstico se basa en una investigación cuidadosa de la historia clínica del paciente para una buena toma de decisiones, la utilidad predictiva de las medidas específicas de diagnóstico, los exámenes radiológicos son necesarios para el análisis del tamaño de la lesión necrótica y planificación del tratamiento (radiografía panorámica, tomografía computarizada, TC de haz cónico, resonancia magnética y gammagrafía ósea), y análisis histológico de la zona afectada para excluir la invasión tumoral $(10,14)$.

Inicialmente, no hay evidencia radiológica y el paciente a menudo no presenta síntomas, el dolor por lo general indica una supuesta infección en el hueso expuesto. Un $25 \%$ a $40 \%$ de los casos de ONJ surge espontáneamente, no relacionada con cualquier trauma particular. En estos casos, la sintomatología inicial más frecuente es una sensación desagradable en la boca (adormecimiento, parestesia y sensación de ardor), con los cambios graduales en la mucosa, avanzando a la formación de úlceras que son lentas de curar (15).

Por otro lado, la extracción de los dientes dolorosos puede aliviar los síntomas, pero con el tiempo conduce a una mayor exposición del hueso en pacientes que 
utilizan bifosfonatos a largo plazo $(16,17)$.

Desde que existe, la ONJ es comúnmente asociada con procedimientos dentales que traumatizan al hueso, por ende, es preferible realizar tratamientos endodóncicos, en vez de una extracción del diente, procedimientos invasivos periodontales o la colocación de implantes dentales. Si estos procedimientos no pueden ser aplazados, el reumatólogo debe dar al dentista o cirujano maxilo-facial clara información sobre pacientes con tratamiento de bisfosfonatos, con el fin de planificar el tratamiento dental a tomarlo en cuenta y poner en práctica medidas preventivas para evitar la ONM (17).

El beneficio / riesgo del tratamiento con bisfosfonatos debe ser discutido de forma individual y, cuando sea necesario y posible, optar por una terapia alternativa para la osteoporosis posmenopáusica (18).

Finalmente, las consideraciones prácticas del tratamiento con bisfosfonatos deben tomar en cuenta 4 parámetros: eficacia, cumplimiento (durante la administración del tratamiento), seguridad y adherencia (grado en que el paciente cumple el tratamiento) (7).

\section{PROTOCOLOS ODONTOLÓGICA:} DE ATENCIÓN

Debido al incremento de la prevalencia de pacientes medicados con Bifosfonatos y la necesidad de tratamiento odontológico Capote y cols (2011) proponen lineamientos basados en la literatura que actúan como medidas preventivas antes y durante el tratamiento con bifosfonatos:

"Antes de iniciar tratamiento con bifosfonatos: Estos pacientes deben ser examinados por el odontólogo clínica y radiográficamente, enfatizando la necesidad de mantener una buena higiene bucal; informar al paciente sobre los riesgos asociados al fármaco; cualquier procedimiento dental quirúrgico debe ser finalizado antes del tratamiento con bifosfonatos; estructuras dentarias con mal pronóstico deben ser extraídas, con por lo menos 3 meses de antelación al inicio del tratamiento con estos fármacos, los servicios de oncología deberían remitir al paciente 3-4 semanas antes de iniciar el tratamiento con bifosfonatos para control odontológico. Durante el tratamiento con bifosfonatos: Mantener un control odontológico de 3 a 6 meses; la limpieza dental de rutina debe realizarse con cuidado, evitando heridas en el tejido blando; las dentaduras removibles deben ser chequeadas por su potencial de inducir trauma; el tratamiento endodóntico es preferido ante la extracción dental; los implantes dentales deben ser evitados; si la cirugía es necesaria debe considerarse la prescripción de antibióticos pre quirúrgicos 72 horas antes y postquirúrgicos por un periodo mínimo de 10 dias; suspensión de la terapia con Bisfosfonatos, 3 meses antes y 3 meses después del tratamiento odontológico quirúrgico; en el caso de los pacientes con terapia oral, estimular la cicatrización por primera intención de todas las heridas en cavidad bucal, en los casos que sea posible, sin levantamiento de colgajo" (6).

El uso de antibióticos para tratar lesiones causadas porONM ha tenido resultados ambiguos sin embargo este va a depender del criterio clínico. La elección del antibiótico será determinada preferiblemente por el estudio de cultura y la prueba de sensibilidad de la lesión. Se ha sugerido que la combinación de amoxicilina y metronidazol puede dar buenos resultados $(3,6)$.

\section{RECOMENDACIONES EN EL TRATAMIENTO ODONTOLÓGICO}

Con respecto a las recomendaciones en el tratamiento odontológico Prado y Cols. nos recomiendan en periodoncia iniciar la terapia básica, si no resuelve se realizará un tratamiento quirúrgico para acceder a las superficies radiculares para facilitar el control de la placa, el recontorneado debe ser mínimo. Debe evaluarse realizar injertos y regeneración tisular guiada los cuales se verán comprometidos por la poca vascularidad (5). En relación con la enfermedad periodontal sólo se extraerán los dientes que presenten movilidad grado 3 o aquellos asociados a un absceso periodontal. En estas situaciones, hay que dar un tratamiento antibiótico adecuado, según la pauta indicada anteriormente (3), 2 días antes del procedimiento (5).

Se debe buscar extracciones atraumáticas con cierre primario de los tejidos antes y después del procedimiento, realizar enjuagues con clorhexidina durante dos semanas, dos veces al día. Profilaxis antibiótica 
en procedimientos complejos, presencia de cofactores para desarrollar OMIB, uso prolongado de BBF, iniciar antibioticoterapia 2 días antes del procedimiento (5). Cuando se tiene que realizar un procedimiento quirúrgico oral en un paciente en tratamiento con BFF hay que tomar unas precauciones especiales como: realizar técnicas estrictamente asépticas, realizar una cirugía atrau $\neg$ mática y lograr un cierre por primera intención cuando sea posible. En los pacientes bajo tratamiento con BFF se recomienda plantear un programa continuado de vigilancia cada 3 o 4 meses con control de placa y medidas higiénicas estric$\operatorname{tas}(5)$.

\section{CONCLUSIONES}

- Una buena anamnesis y un adecuado examen clínico son indispensable para detectar pacientes con trastornos metabólicos asociados a la cavidad oral que podrían influenciar en el éxito del tratamiento.

- Se puede concluir que el bisfosfonato más dañino, es el nitrogenado, y la vía de administración más dañina es la IV. A mayor tiempo de expossición de bisfosfonato mayor daño.

- Existe protocolos de atención previos y durante la utilización de bisfosfonatos en el paciente, es deber del odontólogo general tener el conocimiento de ello.

- El trabajo multidisciplinario y la interconsulta son claves en eléxito de nuestro tratamiento.

\section{CORRESPONDENCIA}

Marco Alarcón Palacios

Las Begonias 102 San Felipe, Jesús María

Lima-Perú.

Correo electrónico: marco.alarcon@upch.pe

\section{REFERENCIAS BIBLIOGRÁFICAS}

1. Arrabal M, Valle F, Jiménez A, López V, Arrabal M, Zuluaga A. Tratamiento de la litiasis renal con bisfosfonatos. Arch. Esp. Urol. 2007;60(7):745-54.
2. Escobar E, López J, Marques M, Chimenos E. Osteonecrosis de los maxilares asociada a bisfosfonatos: revisión sistemática. Av. Odontoestomatol 2007;23(2):91101.

3. Ponte N, Estefania R, Aguirre J. Bisfosfonatos y Patología Oral I. Aspectos generales y preventivos. Med Oral Patol Oral Cir Bucal. 2006;11:396-400.

4. Jaimes M, Oliveira GR, Olate S, Albergaria Barbosa JR. Bifosfonatos asociado a osteonecrosis de los maxilares. Revisión de la literatura. Avances en odontoestomatología 2008;24(3):219-26.

5. Prado N, Ensaldo E, Prado J. Manejo Estomatológico del Paciente en Terapia con Bisfosfonatos. Revista ADM enero-febrero 2011;68(1):8-16.

6. Capote C, Mogensen M, Sánchez M, Cedeño J. Consideraciones en el tratamiento odontológico de pacientes en terapia con bisfosfonatos. Acta Odontológica Venezolana 2011;49(4):1-7.

7. Aapro M, Saad F, Costa L. Optimizing clinical benefits of bisphosphonates in cancer patients with bone metastases.Oncologist 2010;15(11):1147-58.

8. Van den Wyngaert T, Claeys T, Huizing M, Vermorken J, Fossion E. Initial experience with conservative treatment in cancer patients with osteonecrosis of the jaw (ONJ) and predictors of outcome. European Society for Medical Oncology. Febrero 2009;20(2):331-6.

9. Picardo S, Levy D, Rey E. Osteonecrosis maxilar: una problemática odontológica. Revista de la Asociación Médica Argentina. 2011;124(1):26-31.

10.Khan A. Bisphosphonate-associated osteonecrosis of the jaw. FP Watch Surveillance medicale (Canadian Family Physician). 2008;54(1):1019-21.

11.McCauley L, Li X. Distinguishing features of the oral cavity and its predisposition to osteonecrosis. Journal Musculoskeletal Neuronal Interactive 2007;7(4):356-7.

12.American Association of Oral and Maxillofacial Surgeons Position Paper on Bisphosphonate-Related Osteonecrosis of the Jaws. Advisory Task Force on Bisphosphonate-Related Osteonecrosis of the Jaws. J Oral Maxillofac Surg 2009; 65:369.

13. Takaishi Y, Ikeo T, Nakajima Y, Miki T, Fujita T. A pilot case-control study on the alveolar bone density measurement in risk assessment for bisphosphonate-related osteonecrosis of the jaw. Osteoporos Int. 2010;21:815825 .

14.Jacobsen C, Metzler P, Obwegeser J, Zemann W, Graetz KW. Osteopathology of the jaw associated with bone resorption inhibitors: what have we learned in the last 8 years? Swiss Med Wkly. 2012 Jun 26;142:w13605. 
15.Carey J, Palomo L. Bisphosphonates and osteonecrosis of the jaw: Innocent association or significant risk? Cleve Clin J Med. 2008 Dec;75(12):871-9.

16.Sanna G, Zampino MG, Pelosi G, Nolé F, Goldhirsch A. Jaw avascular bone necrosis associated with long-term use of biphosphonates. Ann Oncol. 2005;16(7):1207-8.

17.Errante D, Mannucci N, Bernardi D, Bianco A, Salvagno L. Comment on 'Jaw avascular bone necrosis associated with long-term use of bisphosphonates'. Ann Oncol. 2006;17(2):350-2.

18.Capsoni F, Longhi M, Weinstein R. Bisphosphonate-associated osteonecrosis of the jaw: the rheumatologist's role. Arthritis Res Ther. 2006;8(5):219.

19.Jaimes M, Chaves Netto H, Olate S, Chaves M, Barbosa A. Bisfosfonatos asociado a osteonecrosis de los maxilares. Int J Morphol 2008;26(3):681-8.

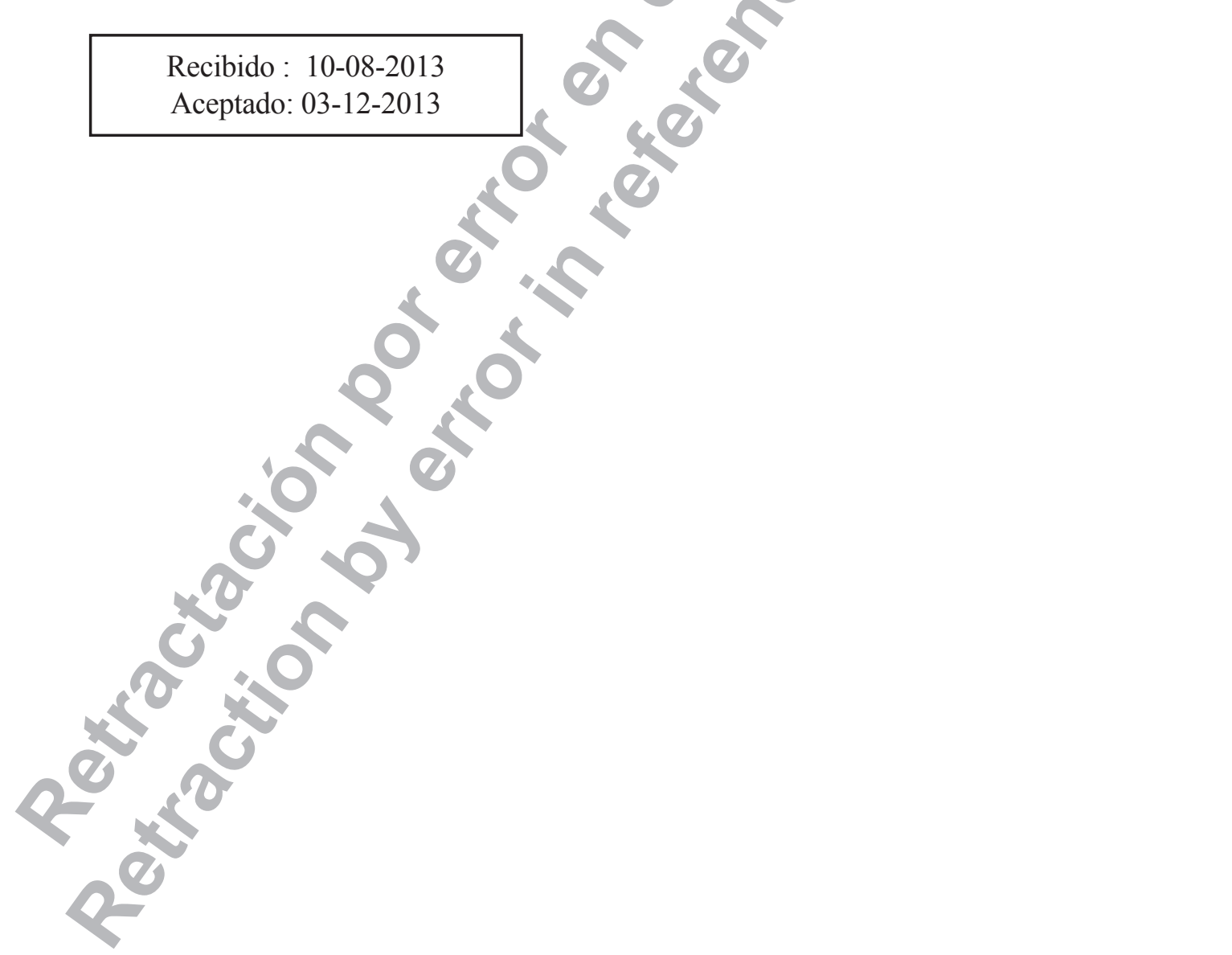

